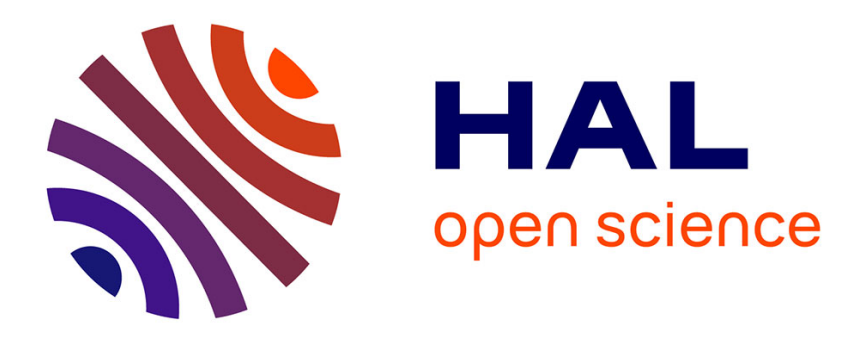

\title{
L'immigration des juifs d'ex-URSS et d'Ethiopie en Israël
}

\author{
William Berthomiere
}

\section{To cite this version:}

William Berthomiere. L'immigration des juifs d'ex-URSS et d'Ethiopie en Israël. Hommes \& migrations, 2002, 1235, pp.40-54. 10.3406/homig.2002.3773 . halshs-01232451

\section{HAL Id: halshs-01232451 https://shs.hal.science/halshs-01232451}

Submitted on 1 Dec 2015

HAL is a multi-disciplinary open access archive for the deposit and dissemination of scientific research documents, whether they are published or not. The documents may come from teaching and research institutions in France or abroad, or from public or private research centers.
L'archive ouverte pluridisciplinaire HAL, est destinée au dépôt et à la diffusion de documents scientifiques de niveau recherche, publiés ou non, émanant des établissements d'enseignement et de recherche français ou étrangers, des laboratoires publics ou privés. 


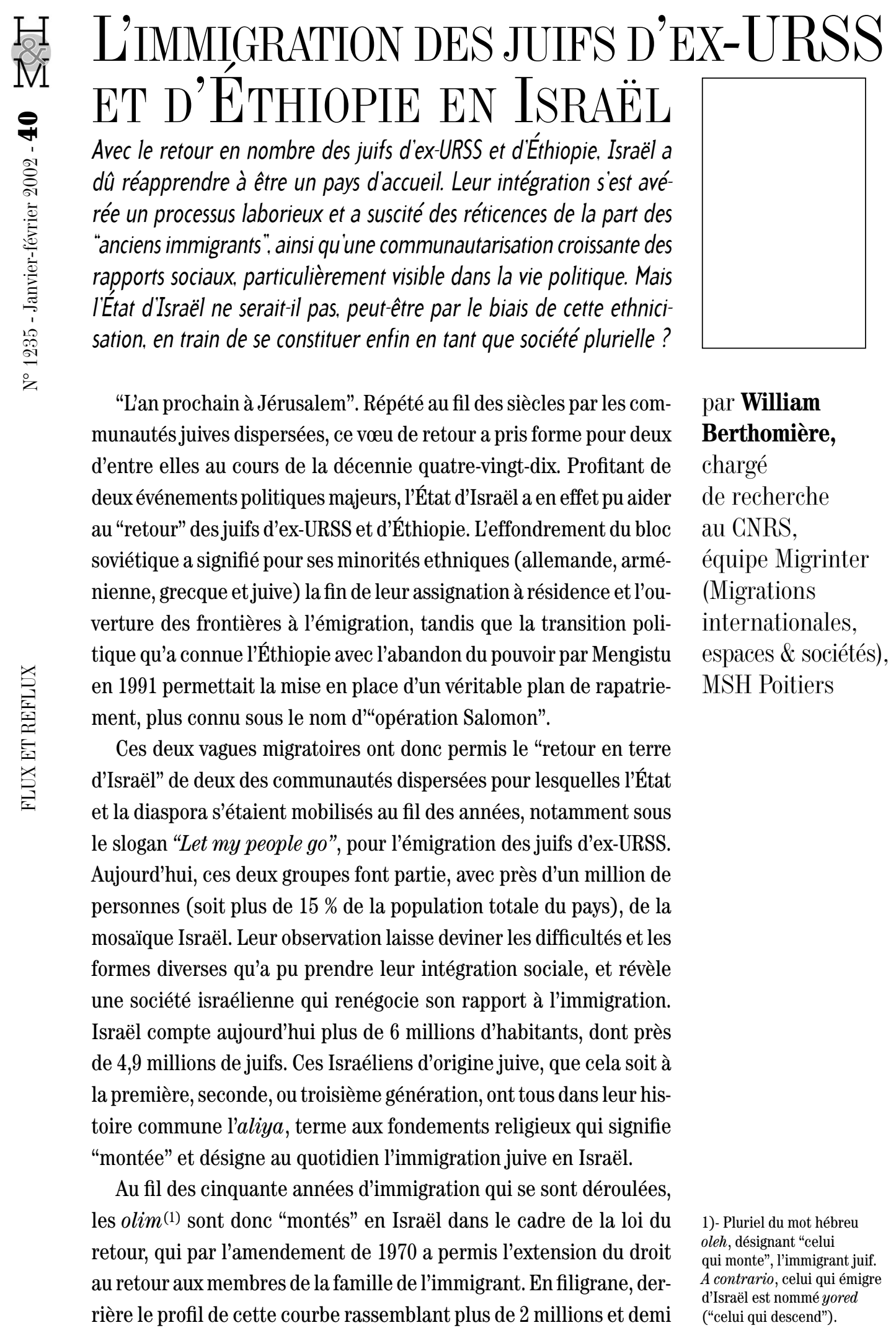


2)- William Berthomière, "De l'aliya à l'immigration ou la lecture d'un continuum migratoire", Revue européenne des migration internationales, spécial Israël, vol. XII, nº 3, 1996 d'immigrants, c'est toute l'histoire de la diaspora juive et de cet État qui transparaît. Dès les premières heures, alors que la première guerre israélo-arabe faisait rage et que plus de 750000 Palestiniens prenaient la route de l'exil, ce sont des milliers de juifs, rescapés de la Shoah mais aussi originaires du Moyen-Orient, comme les juifs d'Irak, qui ont trouvé refuge dans le jeune État. Au cours de ses trois premières années d'existence, Israël, avec plus de 685000 olim, a ainsi doublé sa population. Puis, avec la décolonisation, c'est des rives maghrébines de la Méditerranée que des milliers de juifs sont partis pour Israël. L'immigration marocaine des années cinquante et soixante (270 000 personnes) a constitué la troisième plus importante aliya de l'histoire migratoire de l'État et lui a permis d'approcher les trois millions d'habitants, dont 2435000 juifs( $^{(2)}$.

\section{SE PENSER À NOUVEAU \\ DANS LE TEMPS DU RETOUR}

Au lendemain de ces deux grands événements politiques, l'aliya s'est peu à peu tarie. La croissance démographique d'Israël n'a cessé de s'éroder, passant de $3 \%$ par an sur la décennie 1960 à 1,4 \% par an à la fin des années quatre-vingt. En effet, seuls deux événements sont venus troubler l'histoire migratoire d'Israël après 1965. La guerre des Six-Jours, en faisant rejaillir la crainte d'un nouveau géno-

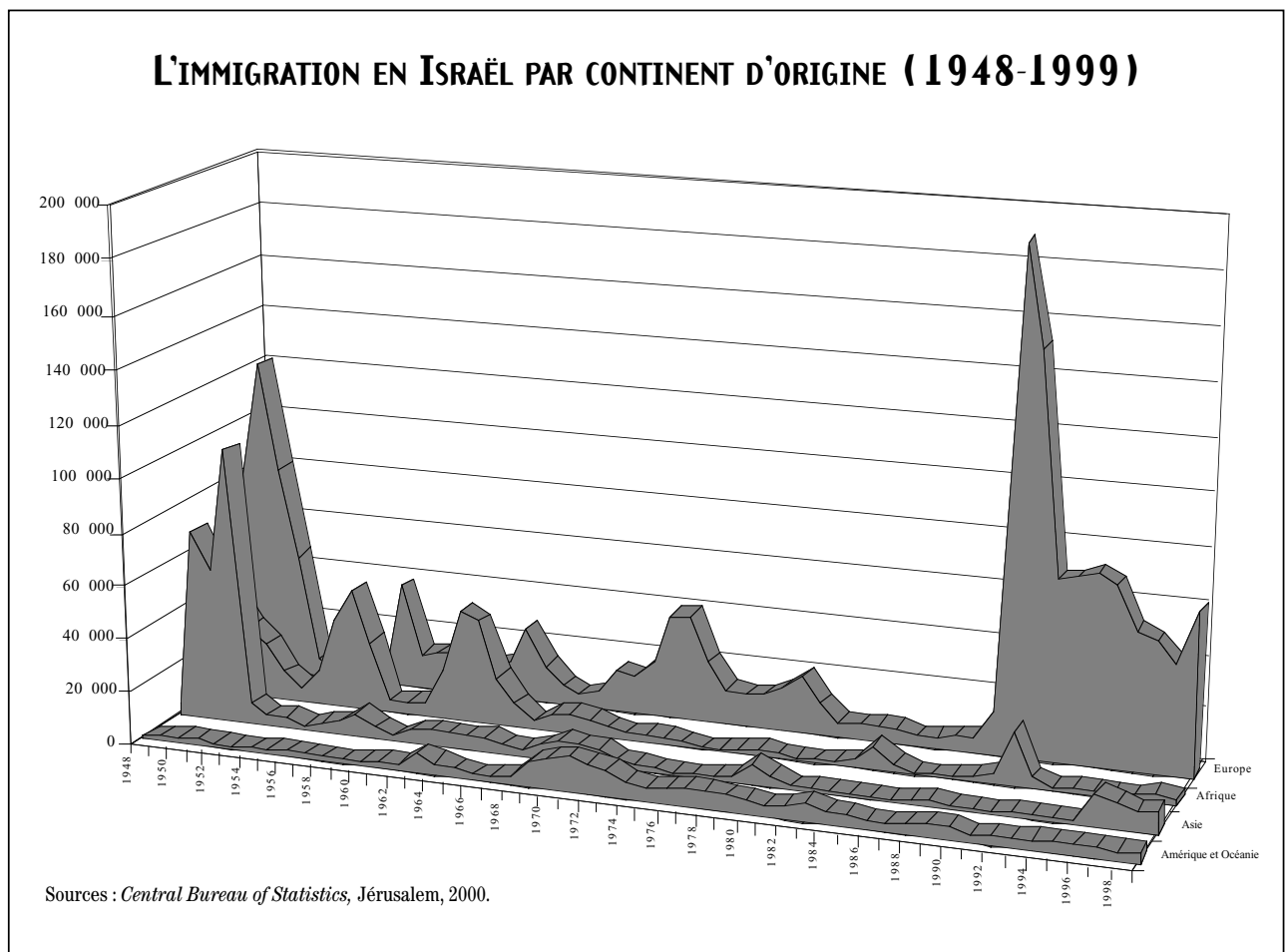


cide a créé un fort mouvement de solidarité au sein de la diaspora, concrétisé par un net sursaut de l'aliya ${ }^{(3)}$. Cette période a d'ailleurs été la seule où les foyers importants de la diaspora ont véritablement participé à l'essor démographique d'Israël. Entre 1965 et 1971, on a enregistré six fois plus d'olim issus des grands établissements de la diaspora (Canada, États-Unis, France, Royaume-Uni) que durant la période 1961-1964 (30 550 contre 4 795). Au total, ces immigrants n'ont représenté que $6,5 \%$ du flux global d'immigration juive enregistré entre 1948 et 1989. Le second événement migratoire a été l'ouverture, plus ou moins grande suivant l'état des relations américanosoviétiques, des frontières de l'URSS à l'émigration juive. Ainsi, entre 1965 et 1989, l'aliya s'est élevée à plus de 400000 personnes, majoritairement des juifs soviétiques.

Plus récemment, les deux événements géopolitiques signalés en introduction sont venus redonner à la courbe de l'aliya le profil de ses premières années, les immigrants d'ex-URSS et d'Éthiopie comptant respectivement pour plus de 850000 et 65000 personnes dans la population israélienne d'aujourd'hui( ${ }^{(4)}$. Avec le retour en nombre des Juifs d'ex-URSS et d'Éthiopie, l'État d'Israël s'est retrouvé, de fait, réinscrit dans une temporalité du retour, alors qu'il s'en était quelque peu distancé. Laccalmie migratoire des années soixante-dix et quatre-vingt, que seuls quelque sursauts d'immigration étaient venus perturber, avait amené à une "distanciation" des rapports entre la diaspora et Israël, ou plus justement à une reformulation de leur relation ${ }^{(5)}$, et avait fait entrer Israël dans une ère sociétale plus introvertie, où se définissaient les contours du "nous, les Israéliens".

Avec l'entrée de plus de 300000 juifs d'Union soviétique et l"opération Salomon" entre 1990 et 1991, Israël a dû réapprendre à être un pays d'accueil et, dans l'urgence, à faire face aux besoins et aux attentes de ces "nouveaux Israéliens". Quelque peu débordé par le nombre des immigrants d'ex-URSS, l'État a mis sur pied un programme dit "d'intégration directe". Chaque immigrant s'est vu attribuer un "panier d'absorption", composé d'un soutien financier pour une année, de détaxes sur une variété de produits et de conditions d'emprunts, notamment pour l'immobilier, à des conditions préférentielles. Une totale liberté quant aux lieux de résidence est laissée aux immigrants munis de ce "pack d'intégration". En cela, cette politique rompt doublement avec les précédentes où les immigrants, lors des premières aliyoth, étaient répartis par l'État sur le territoire et, plus récemment, étaient accueillis dans des merkaz klita ("centres d'absorption") afin de faciliter leur entrée dans la société israélienne. Devant l'importance de la vague d'ex-URSS et le nombre limité de centres, il était impos-
3)- Parallèlement, la guerre des Six-Jours a entraîné à nouveau sur les routes de l'exode plus de 200000 Palestiniens.

4)- Sergio Dellapergola, "The Global Context of Migration to Israel", in Elazar Leshem, Judith T. Shuval (ed.), Immigration to Israel. Sociological perspectives, vol. VIII : Studies of Israeli Society, Transaction Publishers, Londres, 1998 ; Mark Tolts, "Russian Jewish Migration in the Post-Soviet Era", contribution à la Conférence européenne sur la population, La Haye, 1999.

5)- Voir notamment l'étude de Dominique Schnapper sur les juifs de France, Juifs et Israélites, Gallimard, Paris, 1980, p. 162. 
sible de maintenir cette politique d"intégration indirecte", d'autant qu'il avait été décidé de la réserver aux juifs d'Éthiopie. Considérant que ces derniers méritaient une attention particulière, notamment du fait de leur origine rurale, les merkaz klita leur ont été prioritairement ouverts et leur séjour devait y être d'une année maximum.

\section{L'EMPLOI, UNE QUESTION CENTRALE}

Les politiques mises en place n'ont toutefois pas permis d'éviter une déstabilisation du pays dont l'origine se trouve principalement dans un double mouvement. D'une part, la politique du gouvernement Shamir était bien plus tournée vers la réalisation d'un "grand Israël" que vers l'aide à l'intégration des nouveaux arrivants. La politique

\section{DES ACTEURS MALGRÉ EUX DU CONFLIT ISRAÉL0-PALESTINIEN ?}

Se réenracinant dans une pensée nationaliste, les dirigeants israéliens ont pu percevoir ces nouvelles immigrations comme le moyen d'asseoir une politique de maîtrise et de conquête territoriales. Cette représentation de l'immigration, qui a toujours été présente, avec plus ou moins d'intensité, dans les différents gouvernements, est à replacer dans le climat de "guerre démographique" qui règne alors entre Israéliens et Palestiniens. Yasser Arafat déclare même en 1990 : "Je m'oppose par principe à cette immigration; elle risque de renverser le rapport deforce."(1) Après une décennie d'immigration, il semble que le retour à un fort niveau de croissance démographique ait replacé Israël dans une logique de stratégie territoriale telle qu'avait pu la définir David Ben Gourion aux premières heures de l'État, mais il n'a pas été le renfort tant espéré par Yitshak Shamir, que cela soit à l'échelle de la Cisjordanie et de la bande de Gaza, ou à celle de Jérusalem-Est. La présence des immigrants au-delà de la Ligne verte s'explique avant tout par la politique de logement développée par l'État. La mise à disposition, dans les principales implantations de Cisjordanie, de logements largement subventionnés a eu un pouvoir d'attraction bien plus fort que n'importe quelle idéologie de colonisation. Les immigrants, notamment les ex-Soviétiques, qui affrontaient de sévères pertes de statut social, cherchaient à acquérir des logements à moindre coût à proximité des grands bassins d'emploi d'Israël : les implantations de Cisjordanie répondaient à cette attente.

À la fin 2000, pratiquement aucun Éthiopien n'avait choisi de s'installer dans les implantations israéliennes et seulement 15500 ex-Soviétiques (soit $2 \%$ du flux d'immigration) y résidaient : très majoritairement en Cisjordanie et dans ses colonies les plus importantes, la bande de Gaza ne retenant pas leur attention ${ }^{(2)}$.

1)- Philippe Fargues, "Les données démographiques de la paix au Proche-Orient", in L. Blin et Ph. Fargues, L'économie de la paix au Proche-Orient, tome II, Maisonneuve et Larose, Cedej, Paris, 1995, p. 73.

2)- William Berthomière, "L'immigration d'ex-URSS et les colonies de Cisjordanie et de Gaza", Revue européenne

des migrations internationales, vol. XVI, ${ }^{\circ} 3$, pp. 201-216. 
de construction de logements avait été soit reléguée dans le domaine privé (qui ne s'était pas empressé de construire pour les immigrants, ce qui explique la pénurie de logements), soit prise en main par le gouvernement et essentiellement orientée vers les espaces périphériques d'Israël (la Galilée et le désert du Néguev) ou au-delà de la Ligne verte ${ }^{(6)}$.

D'autre part, l'attitude des immigrants dans leur choix de résidence a totalement déséquilibré le pays. Les deux tiers des immigrants avaient élu domicile dans les seuls districts de Tel-Aviv, du Centre et de Haïfa. Les ex-Soviétiques désiraient avant tout résider dans les plus importantes villes d'Israël car ils étaient eux-mêmes originaires de grandes agglomérations et possédaient une forte culture urbaine, et par un tel choix, ils pensaient obtenir plus aisément un emploi. Pour les Éthiopiens, cette question se posait avec moins d'acuité car, après les merkaz klita, un programme d'installation a été mis en place par l'État. Des logements subventionnés à 90 \% leur ont été proposés dans une cinquantaine de communes situées au centre du pays ${ }^{(7)}$. Pour eux comme pour les ex-Soviétiques, la question de l'emploi restait la plus centrale.

Ainsi, le défi le plus important que les gouvernants israéliens eurent à relever est celui de l'emploi. Le problème était d'une réelle amplitude. En 1992, un tiers des immigrants (en âge de travailler) était sans emploi( ${ }^{(8)}$. Le paradoxe de l'intégration de cette vague migratoire fut l'obstacle que constituait la surqualification des migrants. Il est vrai que la lecture des qualifications professionnelles montrait des profils très qualifiés (ingénieurs, médecins, universitaires). Parmi les ex-Soviétiques arrivés entre 1990 et 1995, 40 \% avait effectué treize années et plus d'études (environ $25 \%$ pour la population israélienne). Lintégration professionnelle des migrants était d'autant plus difficile à réaliser qu'il existait une réelle inadéquation entre l'offre et la demande : inadéquation pure et simple liée à l'inutilité des qualifications possédées, dans le cas des ingénieurs en pétrochimie ou en métallurgie par exemple ; inadéquation liée à une saturation du secteur concerné, comme celui de la médecine notamment.

\section{UN ÂPRE PROCESSUS D'INSERTION SOCIO-ÉCONOMIQUE}

Comme dans bien d'autres situations d'immigration, le temps a contribué à gommer les aspérités et les rugosités qui caractérisaient ce processus d'insertion socio-économique au début des années quatre-vingt-dix. Aujourd'hui, les ex-Soviétiques ont un taux de chô-
6)- Cette expression désigne les frontières d'Israël avant les conquêtes territoriales de 1967.

7)- Lisa Anteby, "De nouveaux citoyens à la peau noire : les juifs éthiopiens en Israël", Les Cahiers de l'Orient, $\mathrm{n}^{\circ} 54,1999$, p. 76.

\section{8)- MOIA, Immigrant Absorption: Situation, Challenges and Goals, Ministry of Immigrant Absorption, Planning and Research division, Jerusalem, 1996, p. 17.}


9)- R. Raijman, M. Semyonov, "Best of Times, Worst of Times, and Occupationa Mobility : The Case of the Soviet Immigrants in Israel", International Migration vol. XXXVI, n 3, 1998 .
10)- Anne de Tinguy, "Les Russes d'Israël : l'ethnicité valorisée", Les Cahiers de l'Orient, $\mathrm{n}^{\circ} 54,1999$, p. 55. mage identique à celui des autres Israéliens et ne présentent plus de problèmes de logement. Bien au contraire, ils sont aujourd'hui près de 80 \% à être propriétaires. Ce panorama statistique laisse néanmoins dans l'ombre un processus d'insertion socio-économique qui a pu être très âpre. En effet, pour bon nombre d'entre eux, cette intégration a été synonyme de perte de statut social et d'acceptation d'une politique d'installation en périphérie des grands centres urbains. Pour exemple, l'étude menée par Raijman et Semyonov ${ }^{(9)}$ montre que pour 80 \% des ex-Soviétiques arrivés en 1990, l'entrée sur le marché du travail s'est accompagnée d'un changement d'activité (cette proportion était de $56 \%$ chez ceux venus en 1980) et, pour les deux tiers d'entre eux, elle a signifié une perte de statut social. Cette mobilité professionnelle à la baisse a plus particulièrement touché les femmes : $74 \%$ ont trouvé une activité d'un niveau inférieur à celle occupée avant la migration contre $63,7 \%$ chez les hommes. Le plus fort coût, en termes de perte socio-économique dans la migration, a été payé par les immigrantes et plus particulièrement par celles venues des républiques asiatiques.

Cette perte de statut social, dans bien des cas, s'est accompagnée d'une migration interne vers les périphéries urbaines du pays, là où l'État avait choisi de placer son effort de construction, suivant une idéologie politique où l'aide à l'intégration ne se départait pas de géostratégie. Pour Israël, les résultats affichés sont plutôt flatteurs. La quasi-totalité des ex-Soviétiques ne connait plus de problèmes particuliers ; ils reconnaissent qu'ils choisiraient Israël s'ils devaient à nouveau émigrer ${ }^{(10)}$, tandis que les objectifs d'aménagement du territoire ont connu de véritables succès, le plus remarquable étant d'avoir amené le district Sud (celui du Néguev) au premier rang des districts d'accueil des immigrants. Cependant, si les ex-Soviétiques 
ont pu, après avoir connu une forte déqualification, gravir l'échelle sociale et obtenir des emplois plus proches de leur niveau de qualification, les étapes qui ont jalonné ce processus ont pu marquer les immigrants et les amener à instrumentaliser leur ethnicité. Une telle dynamique a pu voir le jour avec d'autant plus de facilité que les migrations internes opérées ont eu pour effet de favoriser le regroupement communautaire.

Les Éthiopiens ont connu un schéma d'intégration différent, notamment du fait de l'insuffisance de leurs qualifications professionnelles, qui les a cantonBasée sur lidée que l'immigration devait permettre d'assurer l'édification de l'État, la politique d'accueil a consisté à répartir des vagues migratoires dans les espaces sous-peuplés. nés dans des secteurs d'activité peu rémunérés. À la différence des femmes ex-soviétiques, l'immigration a permis une plus grande participation des Éthiopiennes à l'activité professionnelle. Néanmoins, une large part du groupe éthiopien reste dépendant des aides de l'État(11).

\section{DES TENSIONS HÉRITÉES DU PROCESSUS DE CONSTRUCTION NATIONALE}

Au début des années quatre-vingt-dix, le retour à une ère de forte immigration n'a pas constitué, malgré l'effort national que requerrait cet événement, l'occasion pour Israël de renouer avec les valeurs premières du sionisme. La joie suscitée par les vagues migratoires a certes fait rejaillir l'idéal sioniste, mais cette période a été d'assez courte durée. Le pays, préoccupé par le futur de ses rapports avec les Palestiniens, a montré un intérêt modéré envers les nouveaux immigrants, laissant même paraître un certain mécontentement à leur encontre. Ces attitudes plongent leurs racines dans le processus de construction nationale et vont bien au-delà du simple témoignage d'un individualisme grandissant de la société israélienne.

Au cours des premières heures de l'État, la production du projet de société israélien s'est véritablement effectuée dans l'urgence, du fait principalement des conflits armés dans lesquels était engagé le pays. C'est certainement ainsi que les "nouveaux Israéliens" ont su se jouer, ipso facto, de cette question qui intriguait Vladimir Jankélévitch, à savoir "comment les Juifs installés depuis si longtemps dans la langueur de l'absence, pouvaient échapper à l'angoisse des retrouvailles"(12). Si, dans un premier temps, le jeune État a su gouverner et transiger sur le devenir du projet sioniste et, par voie de

11)- Lisa Anteby, "De nouveaux citoyens à la peau noire...", art. cit. p. 78 .

12)- Cité par Marek Halter, Un homme, un cri, Robert Laffont, "Presses Pocket", Paris, 1991, p. 41. conséquence, a su repousser cette "angoisse des retrouvailles", l'agitation sociale grandissante l'a amené à l'affronter. 
13)- Voir les travaux de S. N. Eisenstadt, notamment The Development of the Ethnic Problem in Israeli Society. Observations and Suggestions for Research, The Jerusalem Institute for Israel Studies, Jerusalem, 1986.

14)- Sammy Smooha et Yochanan Peres, cités in Uri Ram, The Changing Agenda of the Israeli Sociology: Theory, Ideology and Identity, State University of New York Press, "SUNY series in Israeli studies", Albany, 1995, p. 99.

15)- Élie Barnavi,

Une histoire moderne d'Israël, Flammarion, Paris, 1988.
Basée sur l'idée que l'immigration était la source de croissance qui devait permettre d'assurer l'édification de l'État et la sécurisation du territoire, la politique d'accueil des immigrants a consisté en la répartition des vagues migratoires dans les espaces sous-peuplés : la Galilée pour le Nord et le Néguev pour le Sud. Cette politique relevait certes du "bon sens", mais elle a achoppé sur le manque de réel soutien socio-économique apporté aux villes composées majoritairement de nouveaux immigrants "déracinés". Le malaise social croissant au sein de ces immigrants s'est progressivement teinté de revendications aux accents ethniques, car la population des villes de développement était presque essentiellement composée de juifs d'Afrique du Nord. Au quotidien, la rudesse des conditions de vie dans ces villes a poussé de nombreux juifs séfarades à quitter leur lieu d'installation pour trouver refuge dans les quartiers déshérités de Jérusalem, de Tel Aviv et de Haïfa. Dans cette dernière ville éclate, en 1959, au sein du quartier de Wadi Salib peuplé de ces familles en perdition, la première révolte majeure des séfarades contre l'Establishment ashkénaze. Ces tensions n'étaient certes pas nouvelles mais avec celles-ci se sont "instituées" les premières zones de fractures, qui vont parcourir la société israélienne tout au long des décennies à venir(13).

\section{LE REPLI DES ANNÉES SOIXANTE-DIX}

Même si la guerre des Six-Jours (1967) a "ressoudé" la société par une rejudaïsation des valeurs israéliennes et a permis un rapprochement avec la diaspora (cf. supra et graphique), les tensions n'en n'ont été que temporairement repoussées. Fin 1970, la situation s'embrase de nouveau avec l'émergence des "Panthères noires", groupe de jeunes qui exprime haut et fort le désarroi des séfarades et la discrimination qu'ils subissent. Un simple regard sur les déséquilibres sociaux de la population reflète aisément les sources de cette crise : une famille ashkénaze percevait en moyenne des revenus 1,4 fois plus élevés que ceux des séfarades (différence d'autant plus sensible que la taille des familles de ces derniers était plus élevée) ; le taux d'accession à l'enseignement supérieur était 6 à 7 fois plus important chez les ashkénazes ; ces derniers étaient à la tête du pouvoir, tandis que les séfarades ne tenaient que des postes secondaires au niveau local(14). Il était d'autant plus difficile à la communauté orientale de faire valoir ses droits qu'elle ne disposait pas d'une élite pour la représenter. La communauté nord-africaine notamment était arrivée sans son leadership, qui avait préféré émigrer en France, aux États-Unis ou au Canada(15). 
Ce climat de tensions, associé au choc de la guerre du Kippour (1973) et à la rudesse des années qui suivirent, ont conduit les séfarades à exprimer leur mécontentent en amenant au pouvoir le Likoud (parti de droite) en $1977^{(16)}$. Un tel choix était lourd de conséquences pour les dirigeants travaillistes - majoritairement ashkénazes - car ce n'était pas un vote pour la droite que venaient d'opérer les séfarades, mais véritablement un vote contre la gauche. Cet acte contestataire exprimait à la fois le rejet de la politique d'"ashkénisation" menée par l'Establishment travailliste, qui niait les particularismes culturels des séfarades, et le désir d'une ascension sociale que l'adhésion à une nouvelle idéologie politique laissait espérer.

Ainsi, ce repli sur lui-même d'Israël qui aurait pu, dans l'esprit des pères fondateurs de l'État, être l'occasion d'une rencontre, celle des immigrants et des fils d'immigrants aux origines culturelles variées, être l'instant propice à une "fusion des exilés" (mizzoug galouyoth), s'est révélé être bien plus déstructurant que fédérateur. Un "second Israël", oriental, a été rendu visible, et au projet de société basé sur la "fusion des exilés" a succédé un modèle pluriculturel de lecture de cette société. Replacé dans ce contexte, les représentations et les attitudes manifestés par la société israélienne à l'encontre de ces "nouveaux Israéliens" peuvent alors être mieux interprétées.

\section{L'AFFAIBLISSEMENT \\ DU RAPPORT À L'ALIYA}

Après l'engouement qu'a suscité notamment le "retour" des Juifs d'ex-URSS, divers événements ont témoigné d'un affaiblissement croissant de l'intérêt israélien pour cette immigration. La "radicalisation ethnique" de la société israélienne semble avoir eu pour

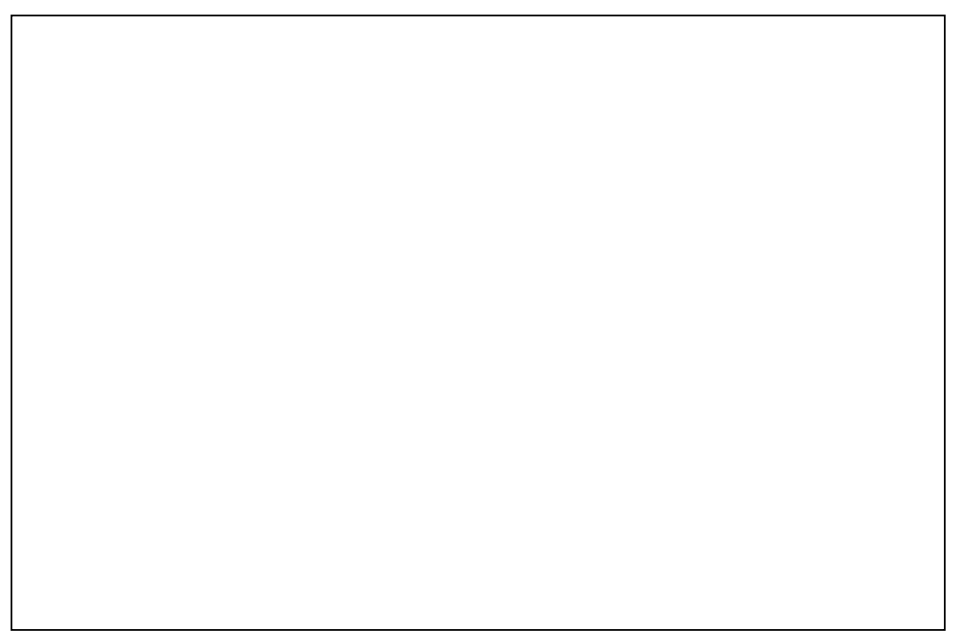

16)- Sur l'analyse de cette période, voir les travaux de Michel Abitbol, notamment

"La mémoire occultée et retrouvée : Juifs d'Orient et de Méditerranée en Israël", in Fl. Heymann (éd.),

Les nouveaux enjeux

de l'historiographie israélienne, CRFJ,

Jérusalem, 1995, pp. 41-49, ainsi que Uri Ram,

The Changing Agenda..., op. cit.
Des immigrants éthopiens manifestent à Beer-Sheva pour protester contre le prix du logement. Les juifs d'Éthiopie ont fait l'expérience d'expressions racistes, comme le refus de certains Israéliens de partager le même immeuble qu'eux. 
conséquence de faire de l'aliya une question bien moins centrale qu'elle ne l'était aux premières heures de l'État. La lecture des sondages concernant la perception de l'immigration a révélé cet état de fait. Sur les deux dernières décennies, le sentiment faisant de l'immigration une valeur centrale d'Israël a été partagé par de moins en moins d'Israéliens : $87 \%$ en 1973 pour seulement $67 \%$ en 1995(17). Plus troublants encore sont les résultats enregistrés sur la période 1990-1994 : si, en début de période, l'intérêt pour l'aliya avait retrouvé une valeur proche de celle de 1973, quatre années plus tard, il avait chuté de plus de $20 \%$.

Dans une perspective "postsioniste",

Le nombre de non-juifs dans l'immigration d'ex-URSS est estimé à environ $30 \%$, et ce sujet a toujours été l'occasion

de tous les abus pour les détracteurs des nouveaux immigrants. cette rapide perte d'intérêt pour l'immigration revêt tous les traits d'une ultime tentative, comme si la population avait vainement tenté de se convaincre - une dernière fois - de la valeur cardinale de l'aliya. Cette sensation est d'autant plus fortement per-

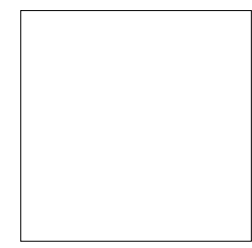

17)- MOIA, Immigrant absorption..., op. cit., p. 57.

18)- Anne de Tinguy, "Les Russes d'Israël, une minorité très influente", Les études du Ceri, $\mathrm{n}^{\circ} 48$, Ceri-Fondation nationale des sciences politiques, Paris, 1998, p. 14.

19)- Anne de Tinguy, "Les Russes d'Israël : l'ethnicité valorisée", art. cité, p. 64 .

20)- Lisa Anteby, "Post-sionisme et aliyah: regards sur l'immigration d'Éthiopie et de l'ex-Union soviétique en Israël", Bulletin du CRFJ automne $1998, \mathrm{n}^{\circ} 3$. ceptible que, dès la fin 1990, une grève générale avait été organisée pour protester contre la levée d'impôts supplémentaires qu'allait devoir supporter la population afin de participer à l'effort national qu'était l'intégration des ex-Soviétiques ${ }^{(18)}$. Même si les fondements de cette distanciation d'avec l'immigration semblent trouver leurs racines dans des considérations essentiellement d'ordre économique, elle a pu être perçue par les ex-Soviétiques comme l'expression d'un rejet. Ce sentiment existait bel et bien car, comme l'a rappelé Anne de Tinguy, un quart des Israéliens déclarait en 1995 ne pas souhaiter un voisinage direct avec les ex-Soviétiques alors qu'ils n'étaient que $4 \%$ en $\left.1990^{(19}\right)$.

Les récents débats autour de la question de la part "grandissante" des non-juifs dans l'immigration d'ex-URSS n'a fait que renforcer le climat de tensions ${ }^{(20)}$. Le nombre de non-juifs dans cette aliya est estimé à environ $30 \%$, et ce sujet a toujours été l'occasion de tous les abus pour les détracteurs du soutien apporté aux nouveaux immigrants. Soulignons que l'immigration d'Éthiopie connaît également un débat similaire. Beaucoup de nouveaux immigrants ont dans leur famille des juifs convertis, nommés falashmoras, et demandent à les faire entrer en Israël. Ce thème soulève d'importants débats, il y aurait aujourd'hui plusieurs milliers de falashmoras en Israël. Les juifs d'Éthiopie ont également fait la triste expérience d'expressions racistes, comme le refus manifesté par certains Israéliens d'accepter que leurs enfants soient à l'école avec 
des Éthiopiens ou de partager le même immeuble qu'eux ${ }^{(21)}$. Pire encore fut la destruction des dons de sang effectués par ces derniers "sur la base de la présence endémique du virus HIV parmi eux"(22).

\section{UNE "ETHNICISATION" CROISSANTE DU DIALOGUE SOCIAL}

Face au mécontentement manifesté par les ex-Soviétiques confrontés aux problèmes d'installation et d'emplois, les vatikim ${ }^{(23)}$ ont été quelque peu choqués, le "second Israël" ressentant comme une injustice les plaintes des nouveaux immigrants ${ }^{(24)}$. Les séfarades vivaient d'autant plus mal les plaintes des ex-Soviétiques que ceuxci n'avaient pas été obligés, comme eux l'avaient été, de s'installer dans des villes périphériques du pays. De surcroît, les nouveaux immigrants qui se sont installés dans les villes de développement, suite aux problèmes d'insertion économique qu'ils rencontraient dans le centre du pays, ont été rapidement perçus comme des concurrents "déloyaux" par nombre de séfarades qui n'avaient pas eu d'autre choix que d'y rester. Très qualifiés et "nantis" par les aides de l'État, ces immigrants ont capté l'attention des employeurs et n'ont fait qu'aviver le sentiment de discrimination des séfarades confrontés au chômage fort élevé de ces villes nouvelles ${ }^{(25)}$.

Sur un plan sociologique, cette première source du rejet manifesté envers l'immigration peut donc s'entendre comme une réification du complexe d'infériorité et du manque de considération fortement intériorisés par les séfarades ${ }^{(26)}$. Ce sentiment était d'autant plus fort que leur situation sociale, en termes d'éducation et de catégories d'emplois, n'avait que peu évolué. Vingt ans après les revendications des Panthères noires, la catégorie des ouvriers rassemblaient plus de $54 \%$ des séfarades et celle des cadres supérieurs seulement $19 \%$; une structure sociale totalement inverse à celle des ashkénazes, où la première catégorie regroupait 28 \% d'entre eux et la seconde 48 \%(27). À la fin des années quatre-vingt-dix, le revenu moyen des séfarades était estimé à moins de 70 \% de ceux des ashkénazes ${ }^{(28)}$. Ainsi, malgré les difficultés socio-économiques que partageait le "Second Israël" avec de nombreux ex-Soviétiques et Éthiopiens, les rapports entre ces populations se sont cristallisés autour de la question ethnique, autour d'une concurrence communautaire.

Contrairement à ce que notait Judith Shuval dans les années quatre-vingt, l'approfondissement des clivages sociaux a évolué vers une "ethnicisation" des partis politiques ${ }^{(29)}$. Si, lors des premières tensions entre ashkénazes et séfarades au cours des années cin-
21)- Lisa Anteby,

"Post-sionisme et aliyah...",

"De nouveaux citoyens

à la peau noire...", art. cit.

22)- Judith Shuval,

"The Sociology of Migration

in Israel: A Critical View",

in Elazar Leshem, Judith

Shuval (ed.), Immigration

to Israel. Sociological

Perspectives, vol. VIII :

Studies of Israeli Society,

Transaction Publishers,

London, 1998, p. 41.

23)- Terme hébreu

signifiant "vétéran", utilisé pour définir les "Israéliens de longue date".

24)- William Berthomière, "L'épopée des Juifs d'ex-URSS en Israël", Confluences Méditerranée, $\mathrm{n}^{\circ} 26,1998$.

25)- R. Isralowitz, I. Abu Saad, "Soviet Immigration: Ethnic Conflicts and Social Cohesion in Israel", International Journal of Group Tensions, vol. XXII, n $2,1992$.

26)- Sur ce thème, cf. Uri Ram The Changing Agenda..., op. cit., chap. VI, "Beyond the Melting Pot: Pluralism", pp. 97-117.

27)- Voir le Jerusalem Report, 12 juin 1997, et l'article de Peter Hirschberg, "Beyond the Rage. A group of Sephardim, successful children of immigrants, want to restructure what they see as Ashkenazi-dominated Israeli society", Jerusalem Report, 14 septembre 1998.

28)- Claude Klein, Israël, État en quête d'identité, Casterman-Giunti, "XXe siècle", Florence, 1999, p. 53.

\section{9)- Judith Shuval,}

"The Structure and Dilemmas of Israeli Pluralism", in B. Kimmerling (ed.), The Israeli State and Society: Boundaries and Frontiers, State University of New York Press, "SUNY series in Israeli studies", Albany, 1989 , p. 224. 
30)- Claude Klein, Israël, État en quête d'identité, op. cit., pp. 59-61.
31)- Dans la perspective communautaire de la société présentée par Uri Ram (op. cit., p. 109), les Arabes d'Israël - et les Palestiniens en général - possèdent le "mode d'incorporation" le plus faible. La politique israélienne développée à leur encontre est perçue comme une domination qui poursuit le but de maintenir ces populations dans leurs frontières et d'exploiter les ressources arabes en faveur de la majorité juive".

32)- Lisa Anteby, "De nouveaux citoyens à la peau noire...", art. cit., p. 85. quante et soixante, les partis de droite et de gauche avaient su coopter les leaders des mouvements de protestation afin d'éviter une "balkanisation" de la politique israélienne, aujourd'hui, ce n'est plus le cas, et les partis "ethniques" sont acteurs de la vie politique israélienne. L'observation de ce processus est d'autant plus intéressante qu'elle indique une véritable complexification des clivages classiques de la société israélienne et révèle les "stratégies sociales" des séfarades.

Comme l'a montré Claude Klein, au clivage classique "droitegauche ou riches-pauvres" qui parcourait la société (juive) israélienne, "traditionnellement composée des Juifs laïques, de droite ou de gauche, ashkénazes ou séfarades (les milieux orthodoxes restant en marge de la société)", ont succédé des clivages superposés articulés autour de deux blocs opposés : le bloc "religieux-séfaradedroite" et le bloc "lä̈que-ashkénaze-gauche", les juifs de gauche étant "désormais plus souvent läques et ashkénazes, ainsi d'ailleurs que de niveau économique plus élevé que les Juifs séfarades, plus pauvres et plus religieux"(30).

\section{DE L'ATTENTISME À L'ACTIVISME POLITIQUE}

Cette relecture des dynamiques communautaires permet de mieux comprendre les rapports de pouvoir qu'elles sous-tendent. Le rapprochement "séfarades-religieux" apparait en effet comme le seul moyen de concurrencer l'intégration et le statut social des ashkénazes ${ }^{(31)}$. Ainsi, un "parti ethnique" comme le Shas, parti séfarade orthodoxe créé en 1984, a pu s'attirer les faveurs des jeunes générations séfarades en leur proposant l'aide sociale et la reconnaissance que ne leur avaient jamais témoignée les travaillistes, ni la droite d'ailleurs. Dès sa première participation, les suffrages exprimés lui ont permis d'obtenir quatre sièges à la Knesset en 1984, et six quatre ans plus tard (cf. tableau p. 52). En prenant des électeurs séfarades au Likoud mais aussi au PNR, le Shas a affirmé "l'ethnicisation" grandissante de la société israélienne.

Face à ce contexte social, les immigrants d'ex-URSS et d'Éthiopie ont répondu différemment. Les Éthiopiens, peut-être du fait de leur faible poids électoral, se sont inscrits dans le débat politique bien plus comme citoyens que comme membres d'une communauté ethnique. Ils ont accordé leurs voix aux partis religieux et au Likoud, et n'ont eu qu'un seul député à la Knesset (le parlement israélien), Adissu Messale, populaire au sein du groupe pour avoir dénoncé le comportement des autorités sanitaires lors de l'affaire des dons de sang(32). 


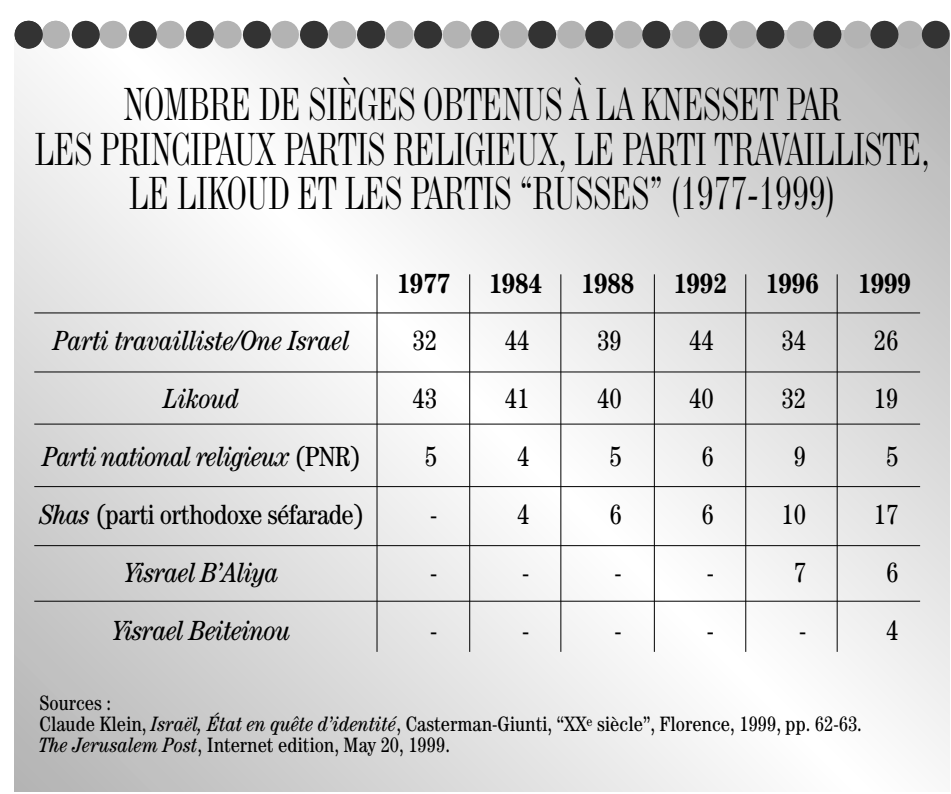

Du côté des ex-Soviétiques, la lecture de la société israélienne s'est faite en deux temps. En premier lieu, les immigrants ont adopté une position attentiste vis-à-vis de l'État, plaçant dans la politique d'intégration les tenants et les aboutissants de leur "communautarisaton". Leur mécontentement - face aux difficultés d'insertion professionnelle notamment-s'est toutefois exprimé par un vote sanction au profit des travaillistes en 1992. Une proposition de création d'un parti russe avait été repoussée par Nathan Sharansky, refuznik et leader charismatique des ex-Soviétiques, et la tentative d'un membre du groupe, Iouli Kocharovsky, faite sous la banderole d'un parti $D a$, s'était soldée par un échec. Cependant, l'attentisme des ex-Soviétiques a progressivement laissé la place à un activisme politique, impulsé par le processus de communautarisation, qui s'est opéré après 1992. La forte perte de statut social et le chômage qui caractérise la situation des ex-Soviétiques arrivés au cours des fortes vagues migratoires des années 1991 et 1992 a mené à un renfermement du groupe sur lui-même.

Du refuge trouvé dans "l'espace du cyrillique", où la communautarisation s'est faite notamment par voie de presse, avec la multiplication des journaux de langue russe, est sorti un groupe ex-soviétique convaincu que l'amélioration de sa condition sociale ne pouvait se faire qu'en empruntant le chemin de la revendication ethnique. Signe d'un réel changement d'objectifs, Nathan Sharansky accepte alors de conduire la liste "russe" aux élections de 1996, qui se nommera Yisrael B'Aliya. Même si une large part du groupe choisit de sanctionner le parti travailliste en ramenant, une fois encore, le parti
33)- Le parti Yisrael Beiteinou ("Israël, notre maison") a été créé par Avigdor Liebermann Ancien conseiller de Benjamin Netanyahou, Liebermann a cherché avec son parti à concurrencer celui de Nathan Sharansky. Soulignons également que lors des élections de 1999, les travaillistes ont créé une coalition regroupant avec eux le parti Gesher et les religieux modérés sous le nom "Un Israël", coalition dirigée par Ehud Barak. 
34)- "Entre citoyenneté et nationalité. Entretiens avec Alain Dieckhoff",

Confluences Méditerranée $\mathrm{n}^{\circ} 26$, été 1998 ;

Alain Dieckhoff, "Israël,

une nation plurielle",

Les Cahiers de l'Orient,

$\mathrm{n}^{\circ} 54,1999$.

35)- Lisa Anteby,

"De nouveaux citoyens

à la peau noire....", art. cit. d'opposition au pouvoir, l'émergence d'un vote ethnique est confirmé avec l'entrée de Sharansky et de six de ses colistiers à la Knesset (cf. tableau). Ils furent renouvelés dans leur mandat aux élections de 1999(33), la permanence du vote "russe" confirmant ainsi l'entrée des ex-Soviétiques dans un schéma ethnique de participation citoyenne.

\section{ISRAËL, SOCIÉTÉ MULTICULTURELLE ?}

Ces dynamiques sociales ont donc considérablement éloigné Israël de ses valeurs fondamentales selon lesquelles le retour des communautés dispersées devait aboutir au mizzoug galouyoth (la "fusion des exilés"). Comme l'a souligné Alain Dieckhoff, la "trop grande épaisseur sociale des différents groupes" a empêché la réalisation du melting pot souhaité par les pères fondateurs de l'État, et la question fondamentale s'articule aujourd'hui autour de la capacité d'Israël à "perpétuer un vouloir vivre ensemble"(34).

Faut-il pour autant poursuivre sur la voie du "pessimisme" amené par le constat d'une "ethnicisation grandissante"? À la lecture des résultats des dernières élections, on ne peut que constater la croissance des partis ethniques.

Face aux difficultés d'intégration rencontrées et au doute émis sur les motivations et sur la judéité de leur aliya par les milieux religieux notamment, l'intelligentsia ex-soviétique a, en réaction, très habilement décrypté le schéma d'organisation sociale du pays en créant $Y i s$ rael B'Aliya. Pour leur part, les juifs éthiopiens, même s'ils font le choix de s'inscrire dans les grands partis, ne négligent pas le "recours" à l'ethnique, notamment sous la forme d'une identification "black"(35). Par ce choix d'instrumentaliser leur ethnicité, les juifs ex-soviétiques, princi-
À Haïfa, le siège du Forum sioniste de Nathan Sharansky, refuznik et fondateur du parti Yisrael B'Aliya. La permanence du vote "russe" confirme l'entrée des ex-Soviétiques dans un schéma de citoyenneté ethnique. 


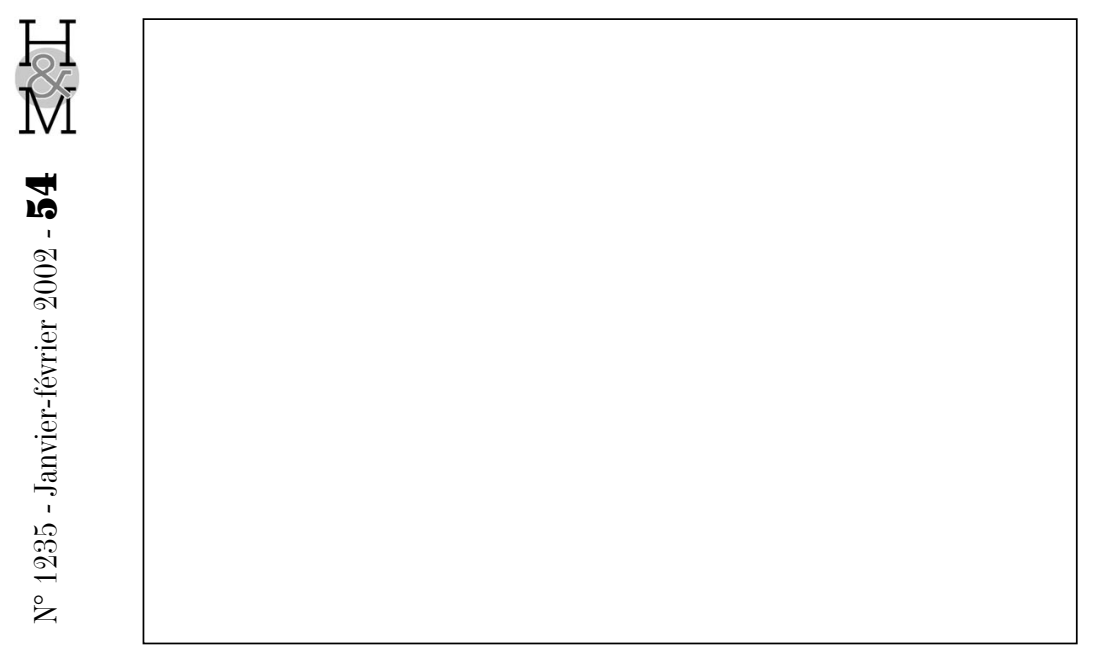

palement, ont contribué au renforcement des clivages, en ralliant le bloc laïc, et démontré que la "communautarisation" constituait, dans cette perspective, la forme la plus efficiente d'expression sociale. Un tel processus renforce donc l'image de la société israélienne proposée par Uri Ram, selon laquelle deux populations s'individualisent : celle qui accorde sa préférence à un État juif (les néosionistes) et celle qui opte pour un État démocratique (les postsionistes)(36).

Cependant, à observer cette société à travers le prisme que constitue le retour des juifs d'Éthiopie et d'ex-URSS, l'idée qu'il s'agit là de véritables structures mais que l'interprétation de leurs interrelations est quelque peu erronée se fait de plus en plus prégnante. En effet, les tensions décrites entre les différents groupes juifs qui composent Israël ne suggèrent-elles pas que la société israélienne opère, à travers ces débats internes, une relecture de son passé au filtre de sa diversité culturelle, plus qu'une "radicalisation ethnique". Ne décidet-elle pas actuellement (seulement maintenant) de son modèle de société ? Différents éléments nous portent à penser que se négocient les bases de l'unité nationale d'une société plurielle telle que peut l'entendre Will Kymlicka(37), caractérisée par sa "polychromie culturelle", pour reprendre l'expression d'Uri Ram(38). Au final, le souhait d'union nationale formulé par Ehud Barak en choisissant de nommer sa coalition "Un Israël" n'était peut-être pas un vœu pieux, juste un peu prématuré...

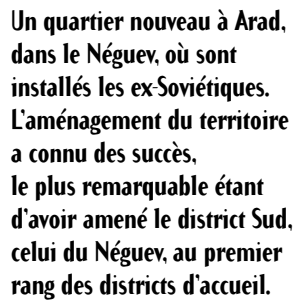

36)- Uri Ram, "Mémoire et identité : sociologie du débat des historiens en Israël", in Florence Heymann, Michel Abitbol, L'historiographie israélienne aujourd'hui, CNRS éditions, CRFJ "Mélanges", Paris, 1998. Selon Uri Ram, le néo-sionisme "représente un mouvement de repli identitaire, nationaliste, raciste et anti-démocratique, qui cherche à élever des barrières autour de l'identité nationale israélienne [...], qui se nourrit de la violence du conflit israélo-arabe et du bas niveau d'intégration dans l'économie capitaliste", et le post-sionisme se présente tel "un courant 'libertaire" d'ouverture qui souhaite réduire les barrières de l'identité nationale et y intégrer l'Autre.

37)- Voir son ouvrage Multicultural Citizenship. A Liberal Theory of Minority Rights, Clarendon Press, Oxford, 1999, traduit en français sous le titre $L a$ citoyenneté multiculturelle : une théorie libérale du droit des minorités, La Découverte, Paris, 2001.

38)- Uri Ram, "Mémoire et identité...", op. cit., p. 229. 